\title{
The effects of the serotonin transporter polymorphism and age on frontal white matter integrity in healthy adult women
}

\author{
Rune Jonassen ${ }^{1,2 *}$, Tor Endestad ${ }^{1}$, Alexander Neumeister ${ }^{3}$, Kari B. Foss Haug ${ }^{4}$, Jens P. Berg ${ }^{4}$ and \\ Nils I. Landrø ${ }^{1}$
}

1 Department of Psychology, Center for the Study of Human Cognition, University of Oslo, Oslo, Norway

2 Division of Psychiatry, Akershus University Hospital, Akershus, Norway

${ }^{3}$ Department of Psychiatry, Mount Sinai School of Medicine, New York University, New York, NY, USA

${ }^{4}$ Section for Research,Department of Medical Biochemistry, Oslo University Hospital, Ullevål, Oslo, Norway

Edited by:

Luiz Pessoa, Brown University, USA

Reviewed by:

David M. Schnyer, University of

Texas, USA

Rebecca Elliott, University of

Manchester, UK

*Correspondence:

Rune Jonassen, Department of Psychology, Center for the Study of Human Cognition, University of Oslo, PO Box 1097, Blindern 0317,

Oslo, Norway.

e-mail: rune.jonassen@psykologi. uio.no
Studies of populations at genetic risk have the potential to explore the underlying structural and functional mechanisms in the development of psychological disorders. The polymorphic region (5-HTTLPR) in the serotonin transporter gene (SLC6A4) has been associated with major depression (MDD) (Caspi et al., 2003). In healthy women, variation in the human brain white matter microstructure integrity in the uncinate fascicule (UF) has been suggested as an endophenotypes in the development of MDD. Pacheco et al. (2009) found a unique effect of age and 5-HTTLPR within the left frontal UF. The present study examined whether these associations persist along the adult life span. Thirty-seven right-handed healthy women between 21 and 61 years of age were invited for a diffusion MRI study. The functional polymorphism 5-HTTLPR located in the promoter region of the SLC6A4 gene was genotyped using polymerase chain reaction (PCR). Fractional anisotropy (FA) was generated for the UF based on Tract-Based Spatial Statistics (TBSS). Models of emotion regulation circuitry suggest that working memory is important in conscious emotion regulation (Price and Drevets, 2010). To explore if 5-HTTLPR is related to this aspects of emotion processing, a working memory pathway, the superior longitudinal fascicule (SLF) was included. The results demonstrate that age may explain the hypothesized association between 5-HTTLPR and frontal UF white matter integrity in healthy adult women. Both white matter changes associated with the aging process and those associated with growth and development may explain why the earlier reported unique effects of genotype in frontal UF FA do not persist into adulthood.

Keywords: white matter integrity, serotonin transporter polymorphism, age, intermediate phenotypes, diffusion tensor imaging

\section{INTRODUCTION}

A unique statistically significant effect of 5-HTTLPR on frontal white matter integrity has recently been reported (Pacheco et al., 2009). Carriers of the short 5-HTTLPR allele showed reduced fractional anisotropy (FA) in the frontal part of the left uncinate fasciculus (UF), a part of a white matter pathway which connects the amygdala to the medial and orbital prefrontal cortex (Pacheco et al., 2009). The study also revealed a unique effect of age. The effects of age on white matter integrity have been studied extensively (Johansen-Berg, 2010). White matter integrity in prefrontal regions increases gradually during adolescence, plateaus during middle adulthood, and degenerates in late adulthood (Giorgio et al., 2010). The sample in Pacheco et al. (2009) consisted of adolescent females and young adults, a restricted age cohort, and did not reveal an age moderated association between FA values and 5-HTTLPR. Another study found that short 5-HTTLPR carriers had decreased functional coupling between the amygdala and the ventromedial prefrontal cortex (Heinz et al., 2005). The few Diffusion Tensor Imaging (DTI) studies on 5-HTTLPR are related to circuitry involved in automatic bottom-up processing of emotion, while there is a paucity of studies on white matter pathways involved in conscious top-down regulation of emotion. The lateral prefrontal cortex (LPF) and the associated white matter pathway, the superior longitudinal fascicule (SLF) is important for conscious emotion regulation (Phillips et al., 2003). The SLF is a thick and long bi-directional bundle of association fibers that extends above the insula from the frontal lobe to the occipital lobe where it curves downward and forward into the temporal lobe. Integrity within SLF has been associated with working memory performance (Karlsgodt et al., 2008). We tested the hypothesis that adult female short 5-HTTLPR carriers have less white matter integrity in the UF compared to long 5-HTTLPR carriers. We also tested the specificity of this association by calculating FA values within the SLF that connect the LPF to the occipital and temporal lobe, a predominantly cognitive pathway. The study develops previous literature by including healthy women covering the whole adult life span. The potential effects of age will, therefore, be of substantial interest as the effects of age are 
among the strongest predictors of FA within the frontal lobes (Westlye et al., 2010).

\section{MATERIALS AND METHODS PARTICIPANTS}

Thirty seven healthy, women, were screened and recruited at the Center for the Study of Human Cognition, University of Oslo to participate in a DTI study. After providing written informed consent, the participants underwent medical and psychiatric evaluations including the Diagnostic Interview for Genetic Studies (Nurnberger et al., 1994), the Structural Clinical Interview for DSM-IV, Axis I, and II disorders (SCID I and SCID II). Depression and anxiety symptoms were assessed using the Beck Depression Inventory (BDI) and the Beck Anxiety Inventory (BAI), respectively. The SCID interviews were collected and recorded by trained clinicians and were subjected to consensus diagnoses. Education level was classified by means of the International Standard Classification of Education (UNESCO, 1997). General cognitive functioning was estimated from scaled scores of two subtests from the WAIS-III, Picture Completion and Similarities (Wechsler, 2003). The Regional Ethics Committee approved the project.

\section{GENOTYPING}

The biallelic 5-HTTLPR polymorphism, located in the regulatory region of the serotonin transporter gene (SLC6A4), was genotyped essentially as described in detail elsewhere (Gelernter et al., 1997a; Stein et al., 2006). A real-time fluorescence Light Cycler instrument was used to amplify genomic DNA by polymerase chain reaction (PCR) in a final volume of $20 \mathrm{ul}$ using Light Cycler FastStart DNA SYBR Green kit (Roche cat no. 12239264001) with specific primers ( $0.5 \mathrm{uM})$ (Gelernter et al., 1997b) generating a long (L) 419 base pair (bp) or a short (S) 375 bp PCR product. Differences in product length depend on the variable number of a $22 \mathrm{bp}$ tandem repeat (VNTR) sequence in the promoter region. Cycle conditions were initiated by 10 min denaturation $\left(95^{\circ} \mathrm{C}\right)$ followed by 45 cycles at $95^{\circ} \mathrm{C}(10 \mathrm{~s}), 66^{\circ} \mathrm{C}(10 \mathrm{~s})$, and $72^{\circ} \mathrm{C}(10 \mathrm{~s})$. Based on the identification of a single nucleotide polymorphism (SNP) within the long variant, suggestions have been put forward that this is a triallelic functional polymorphism (Hu et al., 2006). For the detection of the additional A > G SNP (rs25531), the PCR fragments were digested with $1 \mathrm{U}$ MspI restriction enzyme (New England Biolabs, Beverly, MA) for $2 \mathrm{~h}$ at $37^{\circ} \mathrm{C}$. The PCR fragments contain two obligatory MspI sites, whereas the A > G substitution creates an additional MspI site. The PCR reaction followed by restriction digestion and gel electrophoreses provides classification of the $\mathrm{S}, \mathrm{L}_{\mathrm{A}}$ and, $\mathrm{L}_{\mathrm{G}}$ alleles. The triallelic classification was then reclassified into a biallelic functional model, based on the 5-HTTLPR-directed level of transcriptional activity of the transporter gene as follows: $\mathrm{L}_{\mathrm{G}} / \mathrm{S}, \mathrm{L}_{\mathrm{G}} / \mathrm{L}_{\mathrm{G}}$, and $\mathrm{S} / \mathrm{S}$ genotypes were classified as $\mathrm{S} / \mathrm{S}$ (low-leveled RNA transcription); $\mathrm{L}_{\mathrm{A}} / \mathrm{S}$ and $\mathrm{L}_{\mathrm{A}} / \mathrm{L}_{\mathrm{G}}$ genotypes were classified as $\mathrm{L} / \mathrm{S}$ (intermediateleveled); and $\mathrm{L}_{\mathrm{A}} / \mathrm{L}_{\mathrm{A}}$ genotype was classified as $\mathrm{L} / \mathrm{L}$ (high-leveled) (Neumeister et al., 2006).

\section{DTI ACQUISITION AND ANALYSIS}

Diffusion MRI was acquired on a Philips Archieva 3T MR scanner using echo planar imaging, repetition time/ehco time $=$
$10,793 / 53,2 \times 2 \times 2$ thick (no gap) slices, field of view $240 \times$ $240 \times 120 \mathrm{~mm}$. Sixty slices were acquired, and voxelwise statistical analysis on the FA data was carried out using Tract-Based Spatial Statistics (TBSS) (Smith et al., 2006), a part of FMRIB's Software Library (FSL) (Smith et al., 2004). First, FA images were created by fitting a tensor model to the raw diffusion data using FSL Diffusion Toolbox (FDT), and then brain-extracted using Brain Extraction Tool (BET) (Smith, 2002). All subjects' FA data were then aligned into a common space using the non-linear registration tool FNIRT (Andersson et al., 2007a,b), which uses a b-spline representation of the registration warp field (Rueckert et al., 1999). Next, the mean FA image was created and thinned to create a mean FA skeleton, which represents the centers of all tracts common to the group. Each subject's aligned FA data was then projected into this skeleton and the resulting data fed into cross-subject statistics. The uncinate fascicule (UF) and superior longitudinal fasciculus was defined as a pathway of interest (POI) based on the mean FA skeleton multiplied by the JHU white-matter tractography probabilistic atlas (Hua et al., 2008) in a binary mask (Figure 1). The FSL tool fslmeants was used to create mean FA values from the predefined POI's. The average FA over all voxels in the mask was plotted into Two-Way ANOVAs using Predictive Analytics Software (PASW) Statistics 18 to explore potential effects of genotype within the POI's.

FA values were extracted for each UF section and from the SLF for each hemisphere. Two-Way ANOVAs were conducted to compare FA values for the 5-HTTLPR genotypes. Next, potential effects associated with white matter microstructure development were explored by adding participant age in Two-Way ANCOVAs (analysis of covariance).

\section{RESULTS}

One participant terminated the MR scanning procedure due to scanner-related anxiety. Three participants were excluded based on low quality diffusion weighted images. The remaining 33 participants $(\mathrm{LL}=10, \mathrm{LS}=11, \mathrm{SS}=12)$ were included in the group analysis. Two-Way ANOVAs revealed no statistically significant differences between the 5-HTTLPR genotypes in education level $(M=4.8 \pm 0.8)$, WAIS, PC $(M=13.5 \pm 3.2)$, WAIS,
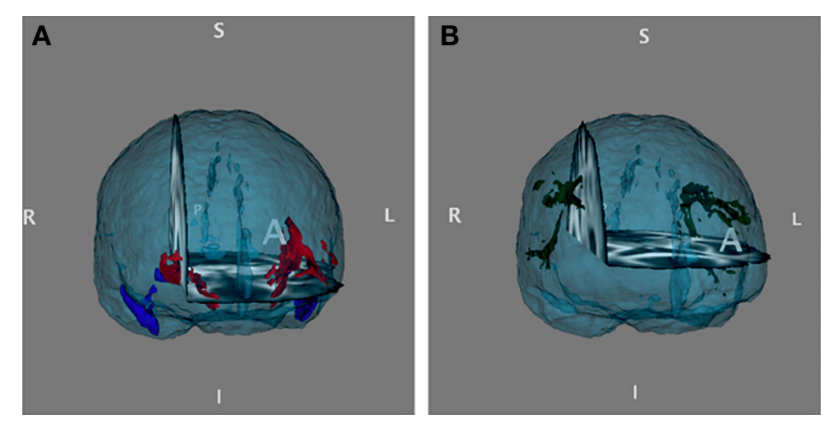

FIGURE 1 | (A) Right and left uncinate fascicule masks and (B) right and left superior longitudinal fascicule, temporal part projected into the mean FA surface, based on the JHU white-matter tractography probabilistic atlas Hua et al. (2008). 
SI $(M=11.0 \pm 2.9)$, BDI $(M=1.6 \pm 2.3)$, or BAI $(M=1.0 \pm$ 1.1). There were no statistically significant differences between the 5-HTTLPR genotypes in age $(M=37.0 \pm 13.1)$. Post hoc LSD tests did not reveal statistically significant age differences between each allele group.

Two-Way ANOVAs revealed a statistically significant effect of genotype in the left frontal UF $\left[F(2,30)=3.3, \eta^{2}=0.18, p<\right.$ 0.047]. There was also a clear trend toward statistically significant effects of genotype in the right $\operatorname{SLF}\left[F(2,30)=3.2, \eta^{2}=0.17\right.$, $p=0.054]$. Polynomial contrast analyses indicated a linear effect for the UF ( $\mathrm{CE}=-0.015, p=0.022)$ but not for the SLF (CE $=$ $-0.007, p=0.129$ ). Despite no statistically significant group differences in age, adding age as a covariate explained the statistically significant effect of genotype in the left frontal UF $[F(1,29)=$ 13.3, $\left.\eta^{2}=0.31 p<0.001\right]$ and the near significant effect of genotype in the right $\operatorname{SLF}\left[F(1,29)=8.6, \eta^{2}=0.22, p=0.008\right]$. The remaining statistics for 5-HTTLPR was $\left[F(2,29)=1.4, \eta^{2}=\right.$ $0.09, p=0.257]$ for the right $\operatorname{SLF}$ and $\left[F(2,29)=1.6, \eta^{2}=0.10\right.$, $p=0.208]$ for the left frontal UF. There was also a main effect of age in the right $\operatorname{SLF}\left[F(1,29)=10.9, \eta^{2}=0.27, p=0.002\right]$. No statistically significant effects of age or genotype were found within temporal parts of the UF or the right frontal UF (Figure 2). Bonferroni corrected alpha levels $(0.05 / 6=0.008)$ did, however, reveal statistically significant local main effects of age in the left frontal UF and right and left SLF.

\section{DISCUSSION}

Despite no statistically significant group differences in age, entering age as a covariate explained the hypothesis of an association between 5-HTTLPR and white matter integrity in healthy adult women. Thus, unlike the previously reported findings in adolescence and young adults, these results did not reveal unique effects of both age and genotype in the left frontal UF. Calculating FA values for a predominately cognitive pathway the SLF, did not reveal a unique effect of genotype after age corrections either.
The results demonstrate that more conservative methodological approaches, where age is controlled for despite no statistically significant differences in age between 5-HTTLPR groups, explain an apparent effect of genotype within the left frontal UF and right SLF.

Other methodological and substantial aspects should be noted. Small sample sizes represent fundamental limitations when testing the association between 5-HTTLPR and age. The pattern of an interaction is also present in our study (Figure 2A). The interaction between age and genotype in the UF may be driven by the older participants. The allele distribution is $\mathrm{LL}=2, \mathrm{LS}=2$, $\mathrm{SS}=6$ in the age cohort $>45$ and $\mathrm{LL}=8, \mathrm{LS}=10, \mathrm{SS}=6$ in the age cohort $<46$. The distributions of alleles in the two age cohorts do not have the power to test this assumption statistically. If the interaction is driven by the older participants it may reflect WM changes associated with the aging process rather that those associated with growth and development. Larger sample sizes are needed to explore this hypothesis of age specific effects.

The 5-HTTLPR genotypes are often treated as a continuous variable (Pacheco et al., 2009). The present study did not reveal linear effect of genotype in the SLF. Assumptions of functional dose effects of genotype, a prerequisite in linear regression analysis, should, therefore, be treated with caution. These analytical approaches also assume that the differences between the three categories (LL, LS, and SS) are the same. Categorical-based approaches, such as ANCOVA used in the present study, are probably more suitable methods to test main effects of age, 5HTTLPR and their potential interaction on FA. Finally, dividing the UF in a frontal and temporal portion is not ideal due to the lack of both anatomical and functional definitions in this region.

There were large local effects of age in the left and right SLF and left frontal UF. The large age span in this study may involve non-linear age effects that increase the variance associated
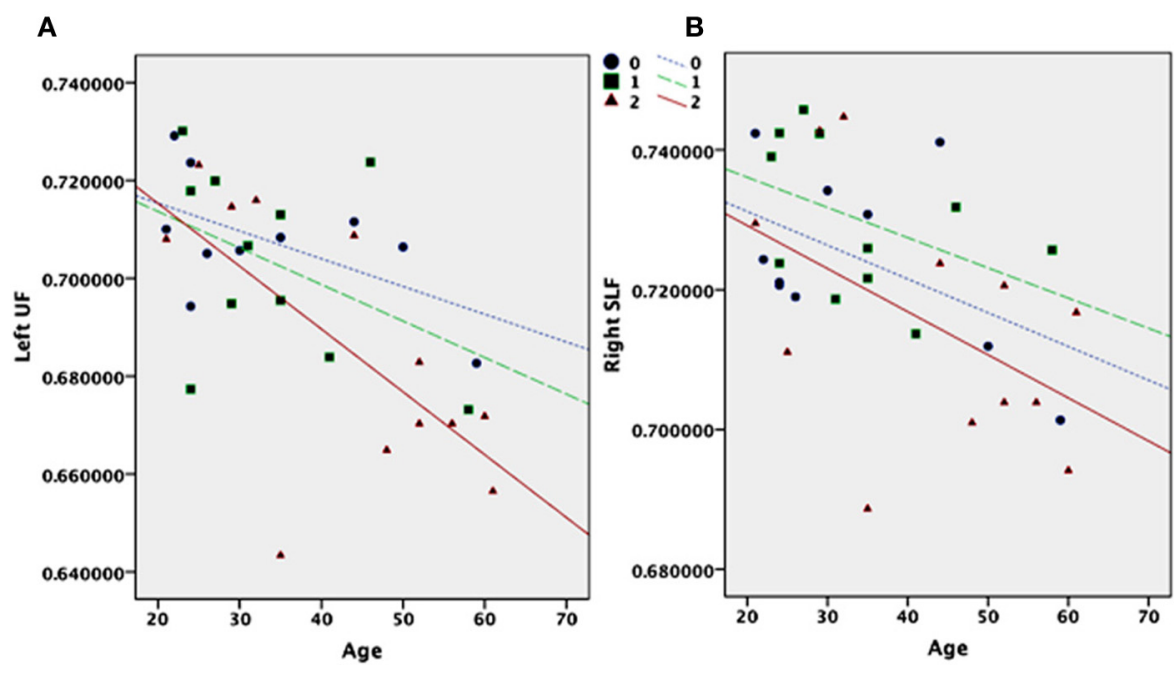

FIGURE 2 | FA values plotted for the number of short 5-HTTLPR copies ( $0=L L ; 1=L S ; 2=S S$ ) based on participants' age for the frontal left UF (A) and the right SLF (B). 
with age (Giorgio et al., 2010). The hypothesis of relatively small 5-HTTLPR effects may be hidden in the background of substantial variance linked to both normal development and the aging process. The association between 5-HTTLPR and frontal white matter integrity may be more present in adolescence since the maturation of human white matter integrity peaks in the twenties (Westlye et al., 2010). Serotonin plays an important role in neural development (Gaspar et al., 2003), but 5-HTTLPR variation may be less significant in normal plasticity or neural degeneration measured by FA. Myelination in the frontal lobes occurs in the adolescence and early adulthood and involves microstructural and biochemical changes at a number of levels (Wozniak and Lim, 2006). The exact relations between the serotonin system and white matter integrity remains speculative but may be particularly associated in periods where the human brain undergoes substantial developmental changes. This may help explain why unique variation linked to serotonin synthesis is absent in the older age cohorts. Together with the study by Pacheco et al. (2009), the two papers cover the whole adult life span. This may indicate that the

\section{REFERENCES}

Andersson, J. L. R., Jenkinson, M., and Smith, S. (2007a). Non-linear Optimisation. [Online]. Available: www.fmrib.ox.ac.uk/analysis/techrep (Accessed 23-08-2011).

Andersson, J. L. R., Jenkinson, M., and Smith, S. (2007b). Nonlinear Registration, aka Spatial Normalisation [Online]. Available: www.fmrib.ox.ac.uk/analysis/techrep (Accessed 23-08-2011).

Caspi, A., Sugden, K., Moffitt, T. E., Taylor, A., Craig, I. W., Harrington, H., Mcclay, J., Mill, J., Martin, J., Braithwaite, A., and Poulton, R. (2003). Influence of life stress on depression: moderation by a polymorphism in the 5-HTT gene. Science 301, 386-389.

Gaspar, P., Cases, O., and Maroteaux, L. (2003). The developmental role of serotonin: news from mouse molecular genetics. Nat. Rev. Neurosci. 4, 1002-1012.

Gelernter, J., Kranzler, H., and Cubells, J. F. (1997a). Serotonin transporter protein (SLC6A4) allele and haplotype frequencies and linkage disequilibria in Africanand European-American and Japanese populations and in alcohol-dependent subjects. Hum. Genet. 101, 243-246.

Gelernter, J., Kranzler, H., and Cubells, J. F. (1997b). Serotonin transporter protein (SLC6A4) allele and haplotype frequencies and linkage disequilibria in Africanand European-American and Japanese populations and in alcohol-dependent subjects. Hum. Genet. 101, 243-246.
Giorgio, A., Watkins, K. E., Chadwick, M., James, S., Winmill, L., Douaud, G., De Stefano, N., Matthews, P. M., Smith, S. M., JohansenBerg, H., and James, A. C. (2010). Longitudinal changes in grey and white matter during adolescence. Neuroimage 49, 94-103.

Heinz, A., Braus, D. F., Smolka, M. N., Wrase, J., Puls, I., Hermann, D., Klein, S., Grusser, S. M., Flor, H., Schumann, G., Mann, K., and Buchel, C. (2005). Amygdalaprefrontal coupling depends on a genetic variation of the serotonin transporter. Nat. Neurosci. 8, 20-21.

Hu, X. Z., Lipsky, R. H., Zhu, G., Akhtar, L. A., Taubman, J., Greenberg, B. D., Xu, K., Arnold, P. D., Richter, M. A., Kennedy, J. L., Murphy, D. L., and Goldman, D. (2006). Serotonin transporter promoter gain-of-function genotypes are linked to obsessive-compulsive disorder. Am. J. Hum. Genet. 78, 815-826.

Hua, K., Zhang, J., Wakana, S., Jiang, H., Li, X., Reich, D. S., Calabresi, P. A., Pekar, J. J., van Zijl, P. C., and Mori, S. (2008). Tract probability maps in stereotaxic spaces: analyses of white matter anatomy and tractspecific quantification. Neuroimage 39, 336-347.

Johansen-Berg, H. (2010). Behavioural relevance of variation in white matter microstructure. Curr. Opin. Neurol. 23, 351-358.

Karlsgodt, K. H., van Erp, T. G., Poldrack, R. A., Bearden, C. E., Nuechterlein, K. H., and

unique effects of genotype in frontal UF white matter integrity do not persist into the whole adult life span. Alternatively, the larger age span from the present study may involve both white matter changes associated with the aging process and those associated with growth and development. Herein, the results from Pacheco et al. (2009) and the present study may reflect two different basal processes.

\section{FINANCIAL DISCLOSURES}

The present research was supported by a grant from the Research Council of Norway to Nils I. Landrø (project number 175387/V50).

\section{ACKNOWLEDGMENTS}

We thank our test assistants Martin Aker, Anne Marie Hoel, and Ragnhild $\mathrm{B} \emptyset$ for their contributions in the screening procedures and Runa M. Grimholt at the Section for Research, Department of Medical Biochemistry, Oslo University Hospital, Ullevål, who performed the genotyping.

Cannon, T. D. (2008). Diffusion tensor imaging of the superior longitudinal fasciculus and working memory in recent-onset schizophrenia. Biol. Psychiatry 63, 512-518.

Neumeister, A., $\mathrm{Hu}, \quad \mathrm{X}$. Z. Luckenbaugh, D. A., Schwarz, M., Nugent, A. C., Bonne, O., Herscovitch, P., Goldman, D., Drevets, W. C., and Charney, D. S. (2006). Differential effects of 5-HTTLPR genotypes on the behavioral and neural responses to tryptophan depletion in patients with major depression and controls. Arch. Gen. Psychiatry 63 978-986.

Nurnberger, J. I. Jr., Blehar, M. C., Kaufmann, C. A., York-Cooler, C. Simpson, S. G., Harkavy-Friedman, J., Severe, J. B., Malaspina, D., and Reich, T. (1994). Diagnostic interview for genetic studies. Rationale, unique features, and training. NIMH Genetics Initiative. Arch. Gen. Psychiatry 51, 849-864.

Pacheco, J., Beevers, C. G., Benavides, C., Mcgeary, J., Stice, E., and Schnyer, D. M. (2009). Frontallimbic white matter pathway associations with the serotonin transporter gene promoter region (5-HTTLPR) polymorphism. J. Neurosci. 29, 6229-6233.

Phillips, M. L., Drevets, W. C., Rauch, S. L., and Lane, R. (2003). Neurobiology of emotion perception II: implications for major psychiatric disorders. Biol. Psychiatry 54, 515-528.

Price, J. L., and Drevets, W. C. (2010). Neurocircuitry of mood disorders.
Neuropsychopharmacology

35 , 192-216.

Rueckert, D., Sonoda, L. I., Hayes, C., Hill, D. L., Leach, M. O. and Hawkes, D. J. (1999). Nonrigid registration using free-form deformations: application to breast MR images. IEEE Trans. Med. Imaging 18, 712-721.

Smith, S. M. (2002). Fast robust automated brain extraction. Hum. Brain Mapp. 17, 143-155.

Smith, S. M., Jenkinson, M., JohansenBerg, H., Rueckert, D., Nichols, T. E., Mackay, C. E., Watkins, K. E., Ciccarelli, O., Cader, M. Z., Matthews, P. M., and Behrens, T. E. (2006). Tract-based spatial statistics: voxelwise analysis of multisubject diffusion data. Neuroimage 31, 1487-1505.

Smith, S. M., Jenkinson, M., Woolrich, M. W., Beckmann, C. F., Behrens, T. E., Johansen-Berg, H., Bannister, P. R., De Luca, M., Drobnjak, I., Flitney, D. E., Niazy, R. K. Saunders, J., Vickers, J., Zhang, Y., De Stefano, N., Brady, J. M., and Matthews, P. M. (2004). Advances in functional and structural MR image analysis and implementation as FSL. Neuroimage 23(Suppl. 1), 208-219.

Stein, M. B., Seedat, S., and Gelernter, J. (2006). Serotonin transporter gene promoter polymorphism predicts SSRI response in generalized social anxiety disorder. Psychopharmacology 187, 68-72.

UNESCO, I. F. S. (1997). International Standard Classification of Education 1997, (ISCED 1997) [Online]. 
Available: www.unescostat.unesco. org (Accessed 23-08-2011).

Wechsler, D. (2003). Wechsler Adult Intelligence Scale - Third edition. Manual. Norwegian version. Stockholm: The Psychological Corporation

Westlye, L. T., Walhovd, K. B., Dale, A. M., Bjornerud, A., Due-Tonnessen, P., Engvig, A., Grydeland, H., Tamnes, C. K., Ostby, Y., and Fjell, A. M. (2010). Life-span changes of the human brain white matter: diffusion tensor imaging (DTI) and volumetry. Cereb. Cortex 20, 2055-2068.

Wozniak, J. R., and Lim, K. O. (2006). Advances in white matter imaging: a review of in vivo magnetic resonance methodologies and their applicability to the study of development and aging. Neurosci. Biobehav. Rev. 30, 762-774.

Conflict of Interest Statement: The authors declare that the research was conducted in the absence of any commercial or financial relationships that could be construed as a potential conflict of interest.

Received: 26 October 2011; accepted: 31 January 2012; published online: 13 February 2012.

Citation: Jonassen $R$, Endestad $T$ Neumeister A, Foss Haug KB, Berg JP and Landrø NI (2012) The effects of the serotonin transporter polymorphism and age on frontal white matter integrity in healthy adult women. Front. Hum. Neurosci. 6:19. doi: 10.3389/fnhum. 2012.00019

Copyright (C) 2012 Jonassen, Endestad, Neumeister, Foss Haug, Berg and Landrø. This is an open-access article distributed under the terms of the Creative Commons Attribution Non Commercial License, which permits non-commercial use, distribution, and reproduction in other forums, provided the original authors and source are credited. 